\title{
Patient Preference between Private and Government Hospital in Hyderabad Region
}

\author{
${ }^{1}$ Dr B Lakshmi, Dr BNV Parthasarathi, Shubham Jain, Monika Bhalerao, Devanshi Saini, Vatsala Mishra, Gaurav Tiwari, Pooja \\ Rathee, Abdul Kareem, Sowmya Nemuri, Janak Varma and Pavan Kuchana
}

\begin{abstract}
Health is most primary factor than money, if people are focused for the treatment of various disorders. As per NSSO report, $72 \%$ and $79 \%$ of rural and urban population using private hospital than government hospital even though private hospital having more cost of treatment than government hospital. Most of people are not using public hospitals mainly due to hygienic condition and low quality of their services. This paper basically deals with the patient perception towards the private and government hospitals in Hyderabad Region. The survey was done using a structured questionnaire for a sample size of 299 outpatients, out of which 130 female and 169 male patients. On basis of this data, we will analyze factor such as demographic, social and economic that responsible for choosing private and government hospital.
\end{abstract}

Keywords:- Private Hospital, Government Hospital, Affordability, Accessibility, Facility, Patient.

\section{INTRODUCTION}

'Health is Wealth' and Good health of population is the 'Wealth of Nation'. After independence of India only $15 \%$ of public was using private hospitals than government hospital. But nowadays, it has been found that about $80 \%$ of the people using the private hospitals than government hospital in India as per World Bank report. As per Boyd report concluded that person having age 19 year or under, $20-44$ and 45 and above age group using 15\%, 59\% and $26 \%$ of public hospitals. Distance of hospitals also one of major factor in preference of hospitals because $41 \%$ of the people went to the nearby hospital. Private hospitals constitute almost $66 \%$ proportion while government hospitals constitute around $31 \%$ of the total hospitals in India. Duggal indicate that private hospital ownership divided into two types i.e., for-profit and not-for-profit and more than $80 \%$ of outpatient care is given by the private sector for the nation.

\section{Public vs private healthcare \\ Private healthcare in India costs about four times more than the public sector, yet majority of all cases are treated by the private sector.}

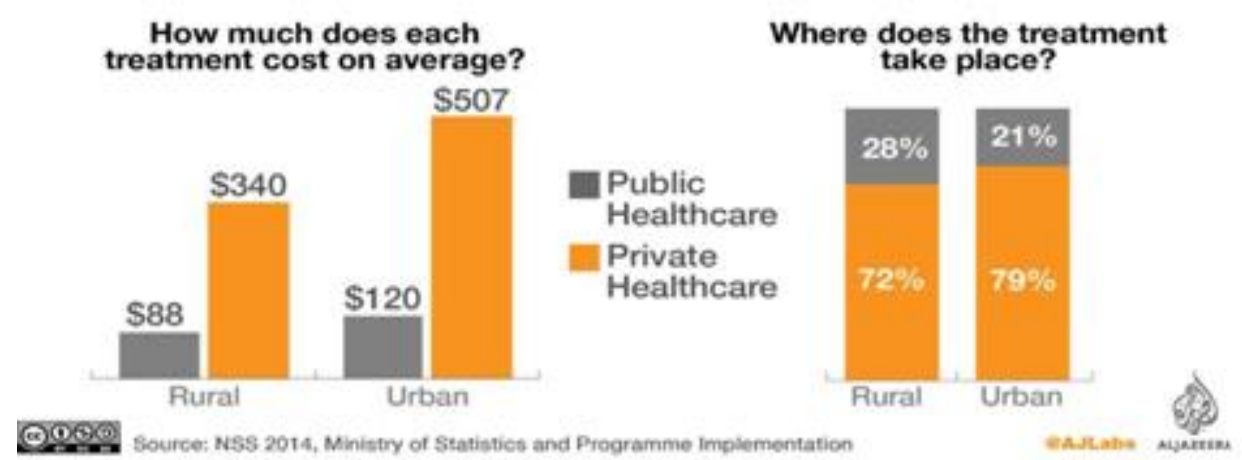

Fig 1

The public hospitals are mostly beneficial for poor and low income household member in society. Even if most of the patients in India using private hospital because public hospital having so much crowd, insufficient doctors and poor hospital services, rude behavior of the medical staff, lack of hygienic conditions etc. On other hand private hospital provides high standard of treatment and hygienic conditions. It is mostly seen that preference of the hospital is affected by social, economic, racial and environmental factors. Government sector typically focused on basic primary health-care, whereas private sector concentrates on secondary and tertiary health services.
The patient preference among hospitals however depends on quality standards of these service providers, self-affordability and accessibility. In conditions of emergency, it is however seen that accessibility becomes a priority rather than affordability whereas for lower income groups affordability is a forever priority. Therefore it becomes important to study patient behavior/preference. Hence, present survey has been run with specific purpose to evaluate the reason behind preference between public and private hospitals for treatment of disorders. 
Through secondary data analysis it was found that there are around 6.1 million populations is residing in Hyderabad (G.H.M.C). To corroborate the findings in the secondary research a module for primary research was designed. Sample size out of the population was found to be 299 which were calculated by using the statistical formulae.

\section{DATA ANALYSIS AND INTERPRETATION:}

Survey Report:

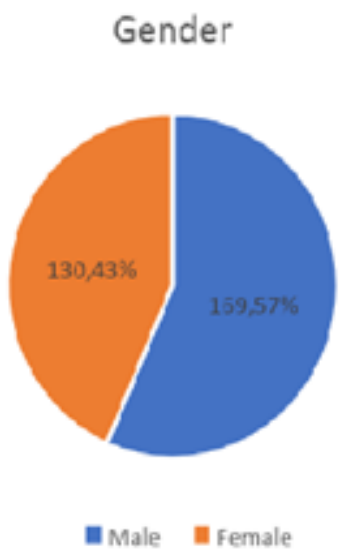

Fig 2

In our study total number of respondents were 299 out of which 130 female and 169 males are their respectively. So, in rate $43 \%$ and $57 \%$ percent were female and male respondent respectively.

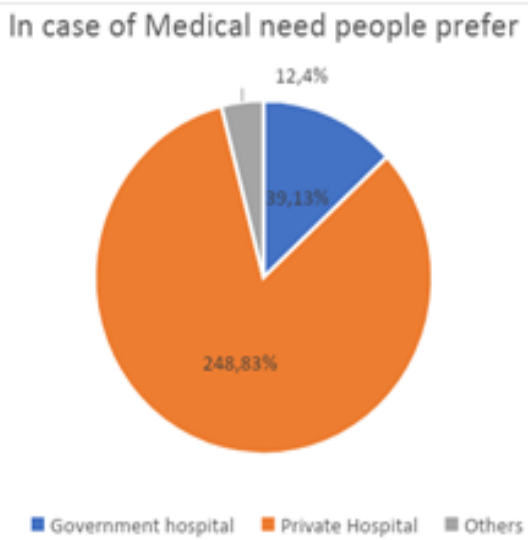

Fig 3

As per review, individual's inclinations for hospitals are $82 \%$ private medical clinic, $13 \%$ government hospitals and $5 \%$ other. So private hospitals ended up selection of individuals when contrasted with govt. and different medical clinics

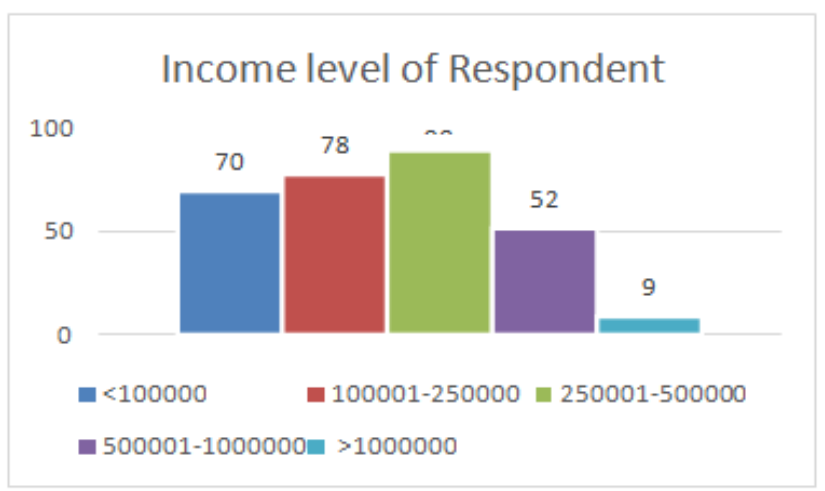

Fig 4

As indicated by pay level 90 respondent were in range of 2.5-5 lakh, 78 were in range of 1-2.5 lakhs, 70 beneath 1 lakhs, 52 were from 5-10 lakhs and 9 respondents were having salary level in excess of 10 lakhs

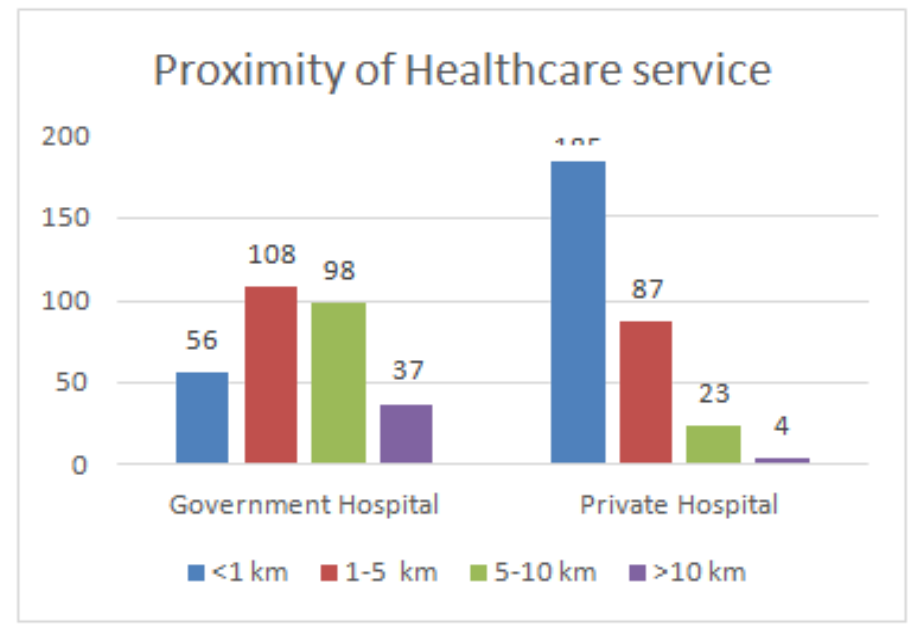

Fig 5

As indicated by vicinity of human services administration government medical clinic was less $1 \mathrm{~km}$ in 56 reactions, $1-5 \mathrm{~km}$ in $108,5-10 \mathrm{~km}$ in 98 more than 10 out of 37 reaction. Were as when we contrast and private clinic there was much distinction as should be obvious, in 185 reactions it is under $1 \mathrm{~km}$, in 85 audit it is $1-5 \mathrm{~km}, 23$ reactions in $5-10 \mathrm{~km}$ territory, were as in only 4 individuals said that it in excess of $10 \mathrm{~km}$ far from their resident. 


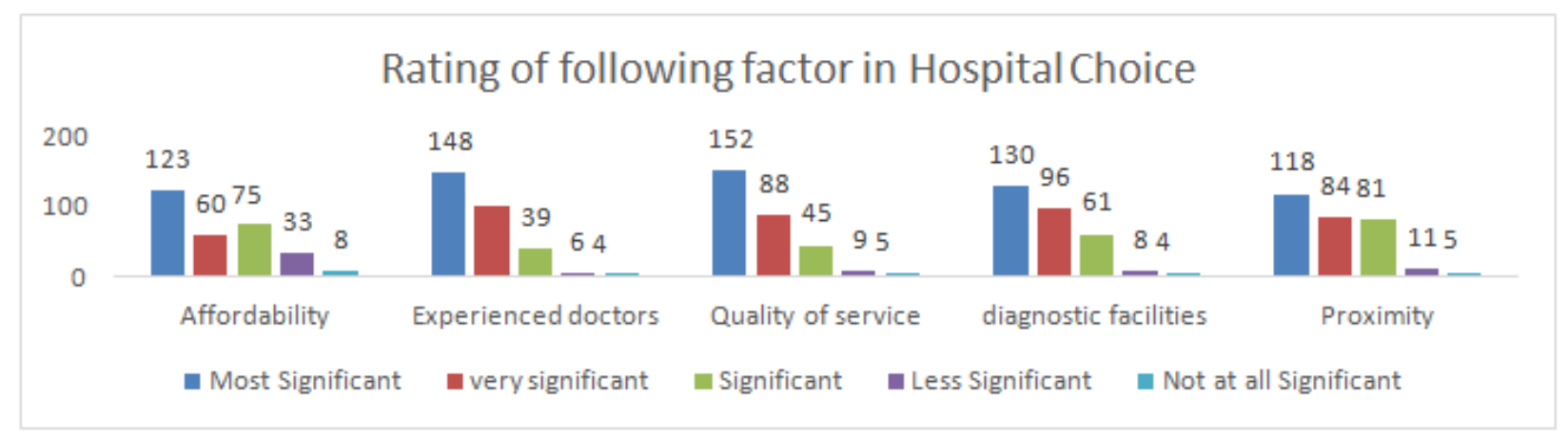

Fig 6

In our survey we have selected 5 factors such as affordability, experience doctor, quality of service, diagnostic facilities and proximity, in determine people preferring hospital. From which $41 \%$ people considered that affordability, $49 \%$ consideration based on experience doctor, quality of services was considered $50 \%, 43 \%$ based on diagnostic center, $37 \%$ relied on proximity from the above responses

Regression Analysis:

a. Dependent Variable: Hospital choice

Model summary

\begin{tabular}{|c|c|c|c|c|}
\hline \multirow{2}{*}{ Model } & $\mathrm{R}$ & R Square & Adjusted R Square & Std. Error of the Estimate \\
\hline 1 & $.173^{\mathrm{a}}$ & .030 & .027 & .353 \\
\hline
\end{tabular}

a. Predictors: (Constant), Affordability

Table 1

\begin{tabular}{|c|c|c|c|c|c|c|}
\hline \multicolumn{7}{|c|}{ ANOVA $^{\mathbf{a}}$} \\
\hline & Model & Sum of Squares & Df & Mean Square & $\mathrm{F}$ & Sig. \\
\hline 1 & Regression & 1.148 & 1 & 1.148 & 9.192 & $003^{b}$ \\
\hline & Residual & 37.080 & 297 & .125 & & \\
\hline & Total & 38.227 & 298 & & & \\
\hline
\end{tabular}

a. $\quad$ Dependent Variable: Hospital choice

b. Predictors: (Constant),Affordability

Table 2

Model summary

\begin{tabular}{|c|c|c|c|c|}
\hline & & & Adjusted R Square & Std. Error of the Estimate \\
\hline 1 & $\mathrm{R}$ & R Square & -.002 & .359 \\
\hline
\end{tabular}

a. Predictors: (Constant), Experienced Doctors

Table 3

ANOVA ${ }^{\text {a }}$

\begin{tabular}{|c|c|c|c|c|c|c|}
\hline \multicolumn{2}{|c|}{ Model } & Sum of Squares & Df & Mean Square & F & Sig. \\
\cline { 1 - 4 } 1 & Regression & .035 & 1 & .035 & .272 & \multirow{2}{*}{$.602^{\mathrm{b}}$} \\
\cline { 1 - 4 } & Residual & 38.192 & 297 & .129 & \\
\hline
\end{tabular}

a. Dependent Variable: Hospital choice

b. Predictors: (Constant),Experienced Doctors

Table 4 
Model summary

\begin{tabular}{|c|c|c|c|c|}
\hline \multirow{2}{*}{ Model } & $\mathrm{R}$ & $\mathrm{R}$ Square & Adjusted R Square & Std. Error of the Estimate \\
\hline 1 & $.132^{\mathrm{a}}$ & .017 & .014 & .356 \\
\hline
\end{tabular}

a. Predictors: (Constant), Quality of service

Table 5

ANOVA $^{\mathbf{a}}$

\begin{tabular}{|c|c|c|c|c|c|c|}
\hline \multicolumn{2}{|c|}{ Model } & Sum of Squares & Df & Mean Square & F & Sig. \\
\cline { 1 - 4 } 1 & Regression & .665 & 1 & .665 & 5.255 & \multirow{2}{*}{$.023^{\mathrm{b}}$} \\
\hline & Residual & 37.563 & 297 & .126 & \\
\hline
\end{tabular}

a. Dependent Variable: Hospital choice

b. Predictors: (Constant), Quality of service

Table 6

Model summary

\begin{tabular}{|c|c|c|c|c|}
\hline & R & R Square & Adjusted R Square & Std. Error of the Estimate \\
\hline 1 & $.073^{\mathrm{a}}$ & .005 & .002 & .358 \\
\hline
\end{tabular}

a. Predictors: (Constant), Diagnostic Facilities

Table 7

ANOVA $^{\mathbf{a}}$

\begin{tabular}{|c|c|c|c|c|c|c|}
\hline \multicolumn{2}{|c|}{ Model } & Sum of Squares & Df & Mean Square & F & Sig. \\
\cline { 1 - 4 } 1 & Regression & .203 & 1 & .203 & 1.585 & \multirow{2}{*}{$.209^{\mathrm{b}}$} \\
\cline { 1 - 4 } & Residual & 38.024 & 297 & .128 & \\
\hline
\end{tabular}

a. Dependent Variable: Hospital choice

b. Predictors: (Constant),Diagnostic Facilities

Table 8

Model summary

\begin{tabular}{|c|c|c|c|c|}
\hline & & & Adjusted R Square & Std. Error of the Estimate \\
\hline 1 & $\mathrm{R}$ & R Square & -.003 & .359 \\
\hline
\end{tabular}

a. Predictors: (Constant), Proximity

Table 9

ANOVA $^{\text {a }}$

\begin{tabular}{|c|c|c|c|c|c|c|}
\hline \multicolumn{2}{|c|}{ Model } & Sum of Squares & Df & Mean Square & F & Sig. \\
\hline 1 & Regression & .003 & 1 & .003 & .027 & \multirow{2}{*}{.870 b } \\
\hline & Residual & 38.224 & 297 & .129 & \\
\hline
\end{tabular}

a. Dependent Variable: Hospital choice

b. Predictors: (Constant),Proximity

Table 10 
- Interpretation for Regression analysis:

For selection of hospital in Hyderabad the preference of people mainly relies on affordability, from regression analysis we can find that the value of $\mathrm{R}$ square for affordability is $3 \%$ which says that the Affordability is significantly important is patient preference for hospital. Similarly, for factors like quality of service and diagnostic equipment availability having R square value of $1.7 \% \& 0.5 \%$ respectively also affects in preference for hospital selection. And factors like Experienced doctor and proximity plays insignificant role in selection of hospital.

ONE-WAY ANOVA:

Hospital choice BY Affordability:

\begin{tabular}{|c|c|c|c|c|c|}
\hline & Sum of Squares & Df & Mean Square & $\mathrm{F}$ & Sig. \\
\hline Between Groups & 1.613 & 4 & .403 & \multirow[t]{3}{*}{3.237} & \multirow[t]{3}{*}{.013} \\
\hline Within Groups & 36.615 & 294 & .125 & & \\
\hline Total & 38.227 & 298 & & & \\
\hline
\end{tabular}

Table 11

Hospital choice BY Experienced Doctors:

\begin{tabular}{|c|c|c|c|c|c|}
\hline & Sum of Squares & Df & Mean Square & F & Sig. \\
\hline Between Groups & .367 & 4 & .092 & .713 & \\
\cline { 1 - 3 } Within Groups & 37.860 & 294 & .129 & & \\
\hline Total & 38.227 & 298 & & \\
\hline
\end{tabular}

Table 12

Hospital choice BY Quality of service:

\begin{tabular}{|c|c|c|c|c|c|}
\hline & Sum of Squares & Df & Mean Square & F & Sig. \\
\hline Between Groups & 1.487 & 4 & .372 & 2.975 & \\
\cline { 1 - 3 } Within Groups & 36.740 & 294 & .125 & & \\
\hline Total & 38.227 & 298 & & \\
\hline
\end{tabular}

Table 13

Hospital choice BY Diagnostic Facilities

\begin{tabular}{|c|c|c|c|c|c|}
\hline & Sum of Squares & Df & Mean Square & $\mathrm{F}$ & Sig. \\
\hline Between Groups & .884 & 4 & .221 & \multirow[t]{3}{*}{1.739} & \multirow[t]{3}{*}{.141} \\
\hline Within Groups & 37.344 & 294 & .127 & & \\
\hline Total & 38.227 & 298 & & & \\
\hline
\end{tabular}

Table 14

Hospital choice BY Proximity:

\begin{tabular}{|c|c|c|c|c|c|c|}
\hline & \multicolumn{2}{|c|}{ Sum of Squares } & Df & Mean Square & F & Sig. \\
\hline Between Groups & \multicolumn{2}{|c|}{.151} & 4 & .038 & .292 & .883 \\
\hline Within Groups & 38.076 & 294 & .130 & & & \\
\hline Total & 38.227 & 298 & & & \\
\hline
\end{tabular}

Table 15

- Interpretation for One-way ANOVA:

When one-way ANOVA is carried out to find the relationship between the dependent variable i.e. people choice between hospital with independent variables like Affordability, Quality of service, Diagnostic equipment availability, Proximity and Experienced Doctors. It was found that the affordability and quality of service were significantly important in their choice of hospital. 
Nonparametric Correlations

VARIABLES= Affordability, Experienced Doctor, Quality of service, Diagnostic Facilities, Proximity, Hospital choice PRINT $=$ SPEARMAN TWOTAILNOSIG MISSING=PAIRWISE

\begin{tabular}{|c|c|c|c|c|c|c|c|}
\hline & & $\begin{array}{c}\text { Affordabilit } \\
\mathrm{y}\end{array}$ & $\begin{array}{l}\text { Experienced } \\
\text { Doctors }\end{array}$ & \begin{tabular}{c|} 
Quality of \\
service
\end{tabular} & $\begin{array}{c}\text { Diagnostic } \\
\text { Facilities }\end{array}$ & Proximity & $\begin{array}{l}\text { Hospital } \\
\text { Choice }\end{array}$ \\
\hline \multirow[t]{18}{*}{$\begin{array}{l}\text { Spearman's } \\
\text { rho }\end{array}$} & \multirow[t]{3}{*}{$\begin{array}{c}\text { Affordability Correlation Coefficient } \\
\text { Sig. (2-tailed) N }\end{array}$} & 1.000 & $.372^{* *}$ & $.328^{* *}$ & $.328^{* *}$ & $.451^{* *}$ & $-.188^{* *}$ \\
\hline & & . & .000 & .000 & .000 & .000 & .001 \\
\hline & & 299 & 299 & 299 & 299 & 299 & 299 \\
\hline & \multirow{3}{*}{$\begin{array}{|rc|}\text { Experienced } & \text { Correlation Coefficient } \\
\text { Doctors } & \text { Sig. (2-tailed) } \\
& \mathrm{N}\end{array}$} & $.372^{* *}$ & 1.000 & $.544^{* *}$ & $.487^{* *}$ & $.457^{* *}$ & .020 \\
\hline & & .000 & . & .000 & .000 & .000 & .731 \\
\hline & & 299 & 299 & 299 & 299 & 299 & 299 \\
\hline & \multirow{3}{*}{\begin{tabular}{|cc}
$\begin{array}{c}\text { Quality of } \\
\text { Service }\end{array}$ & $\begin{array}{c}\text { Correlation Coefficient } \\
\text { Sig. (2-tailed) }\end{array}$ \\
& $\mathrm{N}$
\end{tabular}} & $.328^{* *}$ & $.544^{* *}$ & 1.000 & $.511^{* * *}$ & $.519^{* *}$ & .081 \\
\hline & & .000 & .000 & . & .000 & .000 & .161 \\
\hline & & 299 & 299 & 299 & 299 & 299 & 299 \\
\hline & \multirow{3}{*}{$\begin{array}{|cc|}\text { Diagnostic } & \text { Correlation Coefficient } \\
\text { Facilities } & \text { Sig. (2-tailed) } \\
& \mathrm{N}\end{array}$} & $.328^{* *}$ & $.487^{* *}$ & $.511^{* *}$ & 1.000 & $.583^{* *}$ & .049 \\
\hline & & .000 & .000 & .000 & . & .000 & .399 \\
\hline & & 299 & 299 & 299 & 299 & 299 & 299 \\
\hline & \multirow{3}{*}{$\begin{array}{|cc|}\text { Proximity } & \text { Correlation Coefficient } \\
& \text { Sig. (2-tailed) N }\end{array}$} & $.451^{* *}$ & $.457^{* *}$ & $.519^{* *}$ & $.583^{* *}$ & 1.000 & .000 \\
\hline & & .000 & .000 & .000 & .000 & . & .996 \\
\hline & & 299 & 299 & 299 & 299 & 299 & 299 \\
\hline & \multirow{3}{*}{$\begin{array}{r}\text { Hospital } \\
\text { Choice }\end{array}$} & $-.188^{* *}$ & .020 & .081 & .049 & .000 & 1.000 \\
\hline & & .001 & .731 & .161 & .399 & .996 & . \\
\hline & & 299 & 299 & 299 & 299 & 299 & 299 \\
\hline
\end{tabular}

Table 16

\section{- Interpretation for Correlation study:}

When correlation study is performed between all variables which affects people's preference in their selection of hospital it was found that is no relation between their choice of selection w.r.t Affordability, Experienced Doctors, Quality of service, Diagnostic Facilities, Proximity. It was also observed that affordability and experienced doctor have low to moderate correlation with Quality of service, Diagnostic Facilities, Proximity. Also Quality of service, Diagnostic Facilities and Proximity were internally correlated with one another significantly.

\section{CONCLUSION}

Carrying out the market survey has helped understand the key factor of the patient preferences for any of the hospitals either it be private or government. Thought the cost of private hospitals is high patient prefer it more due to following reasons,

$>$ High standard facilities

$>$ Expert staff

$>$ Clean surrounding

$>$ Proper sanitation of all the belongings

$>$ Hygienic and good quality food available

$>$ Respect given by the doctors and the attending staff

$>$ The atmosphere is maintained lively and keeping the patient away from worrying.
Availability of all the medication.

> The infrastructure and equipment's used are up to mark.

\section{SUGGESTIONS AND RECOMMENDATIONS}

On basis of analysis, we could say that

$>$ Government hospitals supposed to improve hygienic condition and facilities.

$>$ Private hospitals supposed to provide treatment at reasonable and affordable rate, so that low income patients also get advantageous of it.

\section{REFERENCES}

[1]. Dhar A. Activists up in arms against new proposal on health care, 2012. The Hindu. [Aug 09]. p. 13. Back to cited text no. 6

[2]. Prasad S. Morbidity pattern and treatment in India. Ann Trop Med Public Health 2012; 5:459-67.

[3]. White K. The Sage Dictionary of Health and Society. New Delhi: Sage Publication; 2006.

[4]. Irfan, S. M., \& Ijaz, A. (2011). Comparison of service quality between private and public hospitals: Empirical evidences from Pakistan. Journal of Quality and Technology Management, 7(1), 1-22. as accessed on 12th March,2019 at 10:00. 
[5]. Mensah, Francis and Asirifi, Michael Kwabena and Sherif, Mohammed, Comparative Analysis of Patient Satisfaction between Private and Public Hospital (October 2016). American Based Research Journal, Vol-5-Issue-10 Oct-2016 ISSN (2304-7151) as accessed on 13thApril at 11:00.

[6]. Mohd Suki, N., ChiamChwee Lian, J., \&Mohd Suki, N. (2011). Do patients' perceptions exceed their expectations in private healthcare settings? International journal of health care quality assurance, 24(1), 42-56. as accessed on 28th Feb.,2019 at 15:00.

[7]. Narang, R. (2010). Measuring perceived quality of health care services in India. International journal of health care quality assurance, 23(2), 171-186. as accessed on 28th March at 13:00.

[8]. Padma, P., Rajendran, C., \& Sai Lokachari, P. (2010). Service quality and its impact on customer satisfaction in Indian hospitals: Perspectives of patients and their attendants. Benchmarking: An International Journal, 17(6), 807-841. as accessed on 10th March at 14:00.

[9]. Misra Rajiv, Rachel Chatterjee and Sujatha Rao (2003), "India Health Report", Oxford University Press, New Delhi, pp.1-2.

[10]. http://shodhganga.inflibnet.ac.in/bitstream/10603/721 3/14/14_synopsis.pdf as accessed on 16th March at $15: 30$.

[11]. http://www.atmph.org/article.asp?issn=17556783; year $=2013$; volume $=6$; issue $=4 ; \quad$ spage $=472$; epage $=478$; aulast $=$ Prasadas accessed on 1 st March at $13: 30$.

[12]. https://businessjargons.com/spearmans-rankcorrelation-coefficient.htmlas accessed on 15th April at13:00.

[13]. https://math.tutorvista.com/statistics/weightedaverage.html as accessed on 3rdMarch at 11:00.

[14]. https://mohfw.gov.in/sites/default/files/National\%20H ealth $\% 20$ Accounts $\% 20$ Estimates $\%$ 20Report\%202014-15.pdf as accessed on 15th March at 15:00.

[15]. https://newonlinecourses.science.psu.edu/stat501/node /251/as accessed on 20th March at 16:00.

[16]. https://www.cppr.in/wpcontent/uploads/2017/06/Public-and-PrivateHealthcare- Institutions-Preference-and-ExpenditurePattern.pdf As accessed on 25th March at 10:00.

[17]. https://www.hindawi.com/journals/aph/2014/898502/a $\mathrm{s}$ accessed on 10th April at 12:00.

[18]. https://www.ijbssnet.com/journals/Vol_4_No_11_Sep tember_2013/18.pdfas accessed on 15th April at $14: 25$.

[19]. https://www.livemint.com/Politics/1N6EZ3L9TRR9r wj1iocR3L/India-relies-on-private- hospitals-revealsNSSO-survey html as accessed on 20th April at 11:00.

[20]. https://www.statista.com/statistics/684924/indiapublic-health-expenditure/ As accessed on 22nd April at 17:00.

[21]. https://www.technologynetworks.com/informatics/arti cles/one-way-vs-two-way-anova- definitiondifferences-assumptions-and-hypotheses-306553as accessed on 28th April at 18:00. 